\title{
Desarrollo de la Investigación sobre Variación Morfológica de Poblaciones Históricas Sudamericanas Utilizando Rasgos Dentales No Métricos
}

\author{
Development of Research on Morphological Variation of Historical South \\ American Populations Based on Non-metric Dental Traits
}

\author{
Gabriel M. Fonseca,,**; Guillermo Aramburú**; Ivana Rodríguez"**; Gabriel A. Bollini***; \\ Juan P. Atencio***; María J. Berta**; Sandra López-Lázaro*; Mario Cantín ${ }^{* * * * * *}$ \& Rosa G. Lissera**
}

FONSECA, G. M.; ARAMBURÚ, G.; RODRÍGUEZ, I.; BOLLINI, G. A.; ATENCIO, J. P.; BERTA, M. J.; LÓPEZ-LÁZARO, S.; CANTÍN, M. \& LISSERA, R. G. Desarrollo de la investigación sobre variación morfológica de poblaciones históricas Sudamericanas utilizando rasgos dentales no métricos. Int. J. Morphol., 34(1):116-126, 2016.

RESUMEN: El análisis de rasgos no métricos dentales ha logrado establecer relaciones biológicas de grupos humanos pasados y actuales con un alto valor taxonómico. Aunque Sudamérica ha sido objeto de un número considerable de investigaciones sobre poblamiento, migraciones y mestizaje, son relativamente pocos los estudios que han utilizado información de rasgos dentales para este fin, con las consiguiente ausencia de datos en amplias zonas geográficas. Se realizó una revisión sistemática de la literatuara MEDLINE, SciELO, REDALYC y LILACS, sin restricción de fecha de publicación. Se incluyeron artículos completos y disponibles primarios y secundarios en español, inglés y portugués donde se realice el análisis de rasgos morfológicos dentales en poblaciones sudameri canas con un contexto histórico anterior al siglo XX. Los artículos seleccionados fueron evaluados por dos investigadores de maneranidependiente. La búsqueda arrojó 2210 artículos de los cuales 19 cumplieron los criterios de inclusión, a los que se agregaron 9 lueg o de una búsqueda manual complementaria. Existe un desarrollo no equilibrado de la investigación sudamericana, tanto en el foco geográfi co donde esta se realiza, como de los países y filiaciones de sus autoresAunque se han logrado estandarizar los instrumentos devaloración de esos rasgos, se sugiere promover una profesionalización interdisciplinaria, el apoyo internacional de sus proyectos y el abo rdaje holístico de sus contenidos para potenciar la aplicabilidad de su valor taxonómico a poblaciones actuales.

PALABRAS CLAVE: Antropología dental; Rasgos no métricos; Sudamérica; Odontología forense.

\section{INTRODUCCIÓN}

Las reconocidas resistencia y preservación del esmalte dentario han permitido que, en muchos casos, los dientes sean los únicos elementos capaces de ofrecer información biológica individual o poblacional en contextos arqueológicos, pues su morfología se encuentra bajo fuertes controles genéticos (Moreno \& Moreno, 2007; Scott \& Turner II, 2008). En estas situaciones, la antropología dental a través del análisis de cerca de 275 rasgos no métricos dentarios con potencial taxonómico (unos 95 ya convenientemente estandarizados), ha logrado como especialidad de la antropología física establecer relaciones biológicas de grupos humanos pasados y actuales (Rodríguez Flórez, 2003a, 2005; Matsumura \& Hudson, 2005; Moreno \& Moreno). El estudio de la morfología dental ha demostrado poseer alto valor taxonómico favoreciendo la postulación de hipótesis sobre desplazamientos y contactos que han dado lugar al poblamiento y variación étnica de la humanidad (Moreno \& Moreno; Cucina, 2011).

\footnotetext{
* Laboratorio de Pericias en Odontología Forense, Centro de Investigación en Morfología Aplicada, Facultad de Odontología, Uni versidad de La Frontera, Temuco, Chile.

** Facultad de Odontología, Universidad Nacional de Córdoba. Córdoba, Argentina.

*** Facultad de Ciencias Naturales y Museo, Universidad Nacional de La Plata, La Plata, Argentina.

${ }^{* * * * *}$ Centro de Investigación en Ciencias Biomédicas, Universidad Autónoma de Chile, Temuco, Chile.

Trabajo subsidiado por la Secretaría de Ciencia y Tecnología de la Universidad Nacional de Córdoba (S.E.C.y T.), Código de Incentivos 05/J146, resolución Nro. 203/14, Argentina.

Dirección de Investigación, Universidad de La Frontera, Proyecto DIE15-0007.
} 
FONSECA, G. M.; ARAMBURÚ, G.; RODRÍGUEZ, I.; BOLLINI, G. A.; ATENCIO, J. P.; BERTA, M. J.; LÓPEZ-LÁZARO, S.; CANTÍN, M. \& LISSERA, R. G. Desarrollo de la investigación sobre variación morfológica de poblaciones históricas Sudamericanas utilizando rasgos dentales no métricos. Int. J. Morphol., 34(1):116-126, 2016.

Sudamérica ha sido objeto de un número considerable de investigaciones sobre su origen y poblamiento (Bernal \& Luna, 2011). Hanihara definió en 1968 un "complejo dental Mongoloide" basado en sus observaciones sobre cuatro rasgos coronarios en japoneses, indios americanos y esquimales (Huffman, 2014). La mayoría de los estudios han buscado corroborar los tres grandes eventos migratorios de los últimos 20.000 años, cada uno de ellos correspondido estrechamente con los tres grupos de lenguas modernas encontradas entre los nativos de Norteamérica y Sudamérica: Nadene, Eskimo-aleutiano y Paleoindio. Se ha afirmado que las investigaciones de Turner sobre variabilidad de rasgos discretos dentales genéticamente controlados quizás sean "las más completas respecto del origen de la variación biológica humana en el Nuevo Mundo" (Sutter, 2005). Según estos estudios, las poblaciones del noreste asiático y todas las nativas americanas presentan un patrón de rasgos dentales de mayor complejidad ("sinodonte"), evolucionado de uno preexistente más simple ("sundadonte") predominante en poblaciones del sudeste asiático. Estas condiciones dentales han logrado arrojar luz sobre los orígenes genéticos y fenómenos migratorios sudamericanos: para Turner, todos sus nativos se caracterizan por poseer patrones sinodontes relativamente frecuentes (Sutter, 2005).

Rodríguez Flórez (2003a, 2005) afirma que, si bien toda esta información es de gran utilidad para dilucidar y comprender no sólo el poblamiento sudamericano, migraciones, contactos étnicos, niveles de mestizaje actuales e incluso la aplicación de estos conocimientos a contextos de identificación forense, el abordaje holístico para encontrar soluciones comunes a las realidades históricas y a la misma identidad de todos los países involucrados es todavía una tarea pendiente ya que son relativamente pocos los estudios que han utilizado información de rasgos hereditarios dentales para establecer distancias biológicas entre grupos, con la consiguiente ausencia de datos en amplias zonas geográficas y las dificultades implícitas para originar análisis comparativos en Sudamérica.

Considerando que se han sugerido metodologías explícitas para el estudio de la mayor cantidad de rasgos morfológicos dentales con el fin de elaborar comparaciones más consistentes (Turner et al.; Rodríguez Flórez, 2003b) y que la aplicación de estas metodologías observa una evolución desigual en los diferentes países que componen Sudamérica (Rodríguez Cuenca, 1996; Bernal \& Luna), se presenta una revisión sistemática para evaluar la participación de estos países en el estudio de los rasgos morfológicos dentales de poblaciones humanas modernas sudamericanas anteriores al siglo XX, los instrumentos utilizados, y se discuten las posibles implicancias de una contextualización biológica, social y forense de esos estudios.

\section{MATERIAL Y MÉTODO}

Se realizó una revisión sistemática de la literatura en las bases de datos MEDLINE, SciELO, REDAL YC y LILACS, utilizando los términos ("teeth" OR "dental")AND "anthropology" AND ("morphology" OR "phylogeny" OR "trait") en español e inglés con sendas estrategias específicas de búsqueda. Estas se realizaron con fecha 11 al 16 de Octubre de 2015, sin restricción de fecha de publicación. La búsqueda fue realizada de manera simultánea por dos investigadores en forma independiente incluyendo artículos completos y disponibles primarios y secundarios en español, inglés y portugués donde se realice el análisis de rasgos morfológicos externos dentales en poblaciones humanas modernas de asentamiento en Sudamérica con un contexto histórico anterior al siglo XX. Se consideraron como categorías de estudio la nacionalidad de los autores, los países muestreados, el tamaño muestral, el instrumento para el análisis morfológico, la cantidad de rasgos dentales utilizados y los resultados a partir de la obtención de frecuencias absolutas o relativas de los rasgos analizados, la inferencia de procesos microevolutivos asociados a pautas de poblamiento o la discriminación taxonómica a partir de esos resultados. Fueron excluidos estudios que no especificaran los rasgos morfológicos analizados o aquellos que citaran estudios previos si se contaba con el estudio primario correspondiente. Los artículos seleccionados fueron evaluados en su calidad siguiendo la guía de evaluación crítica de la JAMApor dos investigadores de manera independiente, resolviéndose por consenso aquellos casos donde existieron discrepancias.

\section{RESULTADOS}

La búsqueda arrojó 2210 artículos de los cuales 19 cumplieron la totalidad de los criterios de inclusión y presentaron la calidad metodológica necesaria según los estándares descritos. Una búsqueda manual posterior per mitió incluir nueve artículos más que reunían similares criterios resultando en un total de 28 artículos (Fig. 1).

Países de los autores.Argentina y Colombia estuvieron presentes en 12 artículos cada uno, Estados Unidos en seis, Japón en dos, mientras que Chile, España, Perú, Reino Unido y Venezuela en un artículo, respectivamente.Asimismo, resulta indispensable mencionar que los 28 artículos han sido publicados por 36 autores entre los que se destacan Colantonio y Rodríguez Flórez (con respectivos 12 artículos), y Bollini (con ocho artículos); lejos de estos números, pero de presencia significativa, se encontraron Devoto (tres artículos), Turner y Sutter (dos artículos) y Hanihara y Scott, 
FONSECA, G. M.; ARAMBURÚ, G.; RODRÍGUEZ, I.; BOLLINI, G. A.; ATENCIO, J. P.; BERTA, M. J.; LÓPEZ-LÁZARO, S.; CANTÍN, M. \& LISSERA, R. G. Desarrollo de la investigación sobre variación morfológica de poblaciones históricas Sudamericanas utilizando rasgos dentales no métricos. Int. J. Morphol., 34(1):116-126, 2016.

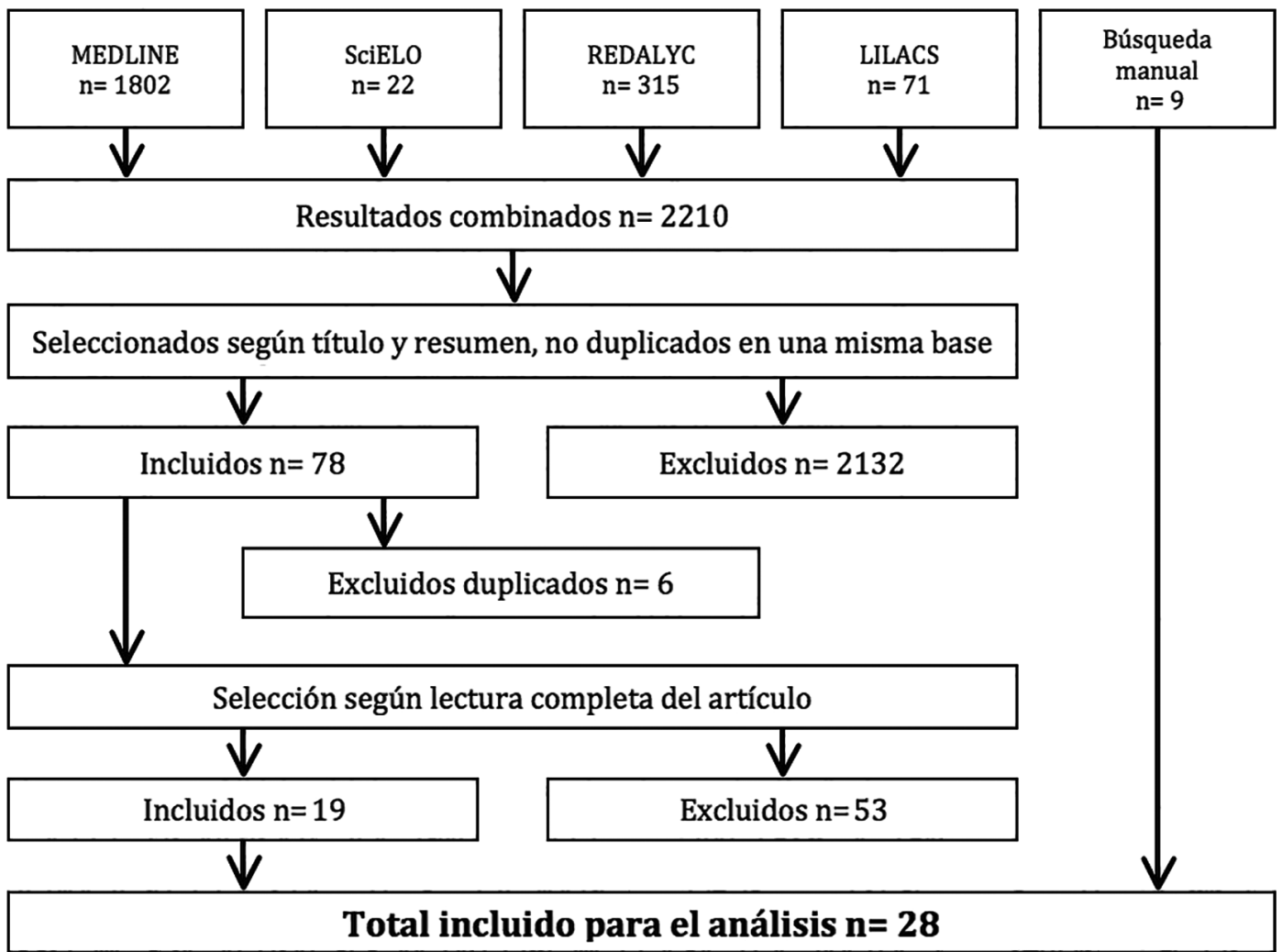

Fig. 1. Flujograma de la revisión de literatura y criterios para selección y exclusión de artículos.

estos último entre los que reportaron un artículo. De los 12 artículos publicados en colaboración internacional, 10 lo fueron entre Argentina-Colombia (iniciada en 2006 con regularidad cercana a la anual, y representada en siete oportunidades casi exclusivamente por el trinomio BolliniRodríguez Flórez-Colatonio), uno entre Venezuela-España (2008) y uno Argentina-Estados Unidos (en 2010). Estados Unidos estuvo representado con un total de 11 autores, Argentina con ocho, y Chile y Venezuela con cinco. Perú lo hizo con filiación de los Estados Unidos (Tabla I).

Países y tamaños muestrales. Argentina representó el mayor número de poblaciones estudiadas con 12 artículos, seguida por Chile con nueve, Colombia con siete, Perú con seis, Venezuela con cuatro, Ecuador y Bolivia con dos, Brasil y Guyana con uno (Thbla I). Sin embargo, analizando los tamaños muestrales según su distribución geográfica, puede observarse en la Figura 2 una clara atención a Colombia, Perú y Chile, incluso con trabajos multinacionales globales (Matsumura, 1995; Hanihara, 2008; Delgado-Burbanoet al., 2010). Los rangos muestrales para las poblaciones sudamericanas estudiadas fueron de siete (Bollini et al., 2009a) a 921 individuos (Delgado-Burbanoet al.). Reyes et al. (2008) y Pomeroy (2009) realizaron reportes de un caso en Venezuela y Argentina respectivamente (Tabla I).
Instrumento para el análisis morfológico y rasgos analizados. El método ASUDAS (Arizona State University Dental Anthropology System) propuesto por Turner et al. (1991) complementado con otros autores según los rasgos morfológicos dentales analizados, ha sido el instrumento utilizado por excelencia para el registro y valoración de los mismos, excepciones hechas de los artículos publicados de manera previa (Devoto \& Arias, 1967; Devoto, 1971; Devoto \& Perroto, 1972; Campusano et al., 1972; Turner \& Bird, 1981) y del reporte de caso de Pomeroy. Sobre este último, vale destacar que el autor refiere al rasgo con el nombre de "T alón Cuspídeo Labial", diferente a la designación estándar ASUDAS de "Tuberculum dentale" (Turner et al.), homologado para esta revisión por Hernández et al. (2010) (Tabla I).

Respecto a la cantidad de rasgos analizados, destacan Rodríguez Flórez \& Pulgarín (2011) quienes analizan un total de 165, y Rodríguez Flórez \& Colantonio (2015) la cantidad de 87, ambos estudios sobre poblaciones colombianas. Atención específica tuvieron los rasgos Reducción del Hipocono (Bollini et al., 2012), Premolar Uto-Azteca (Reyes et al.; Delgado-Burbano et al.; Johnson et al., 2011) y Tuberculum dentale (Pomeroy) (Tabla I). 
FONSECA, G. M.; ARAMBURÚ, G.; RODRÍGUEZ, I.; BOLLINI, G. A.; ATENCIO, J. P.; BERTA, M. J.; LÓPEZ-LÁZARO, S.; CANTÍN, M. \& LISSERA, R. G. Desarrollo de la investigación sobre variación morfológica de poblaciones históricas Sudamericanas utilizando rasgos dentales no métricos. Int. J. Morphol., 34(1):116-126, 2016.

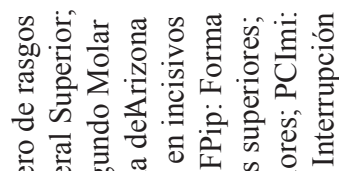

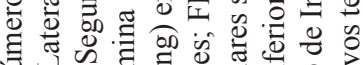

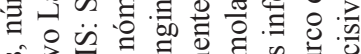

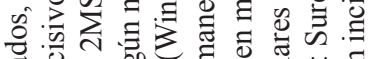

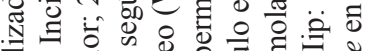

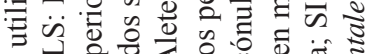

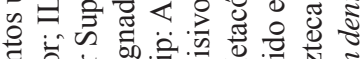

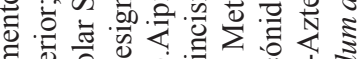

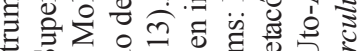

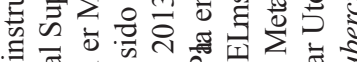

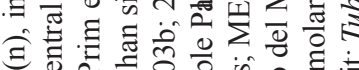
फ 0 论

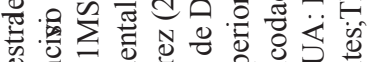

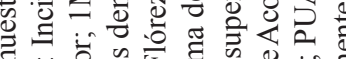

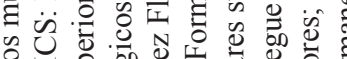

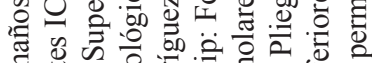

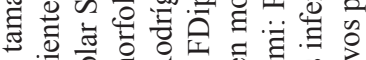
की

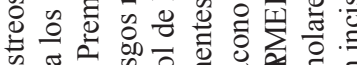
证

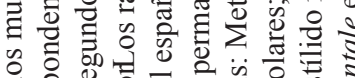
응

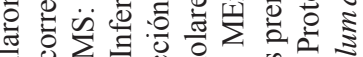
합.

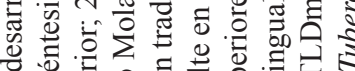

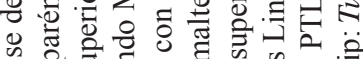

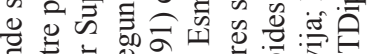

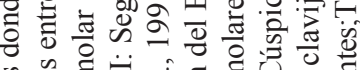

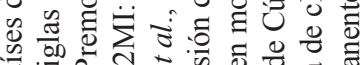

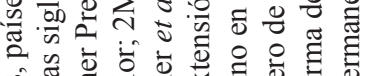

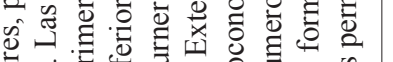

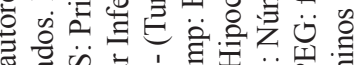

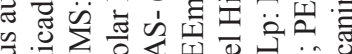

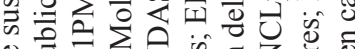
\&

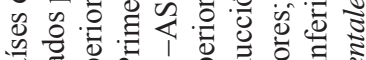

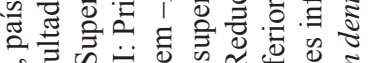

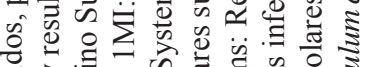

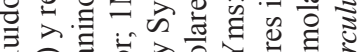

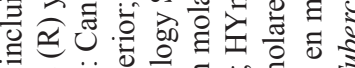

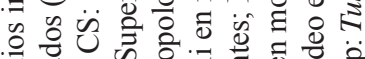

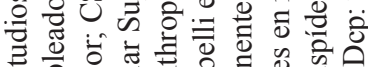

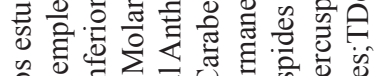

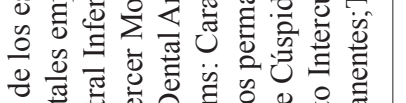

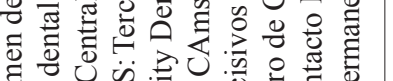

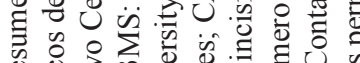

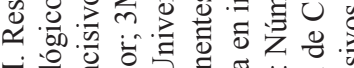

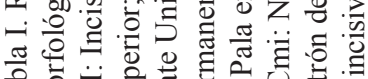

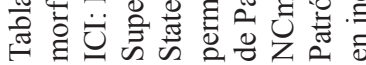
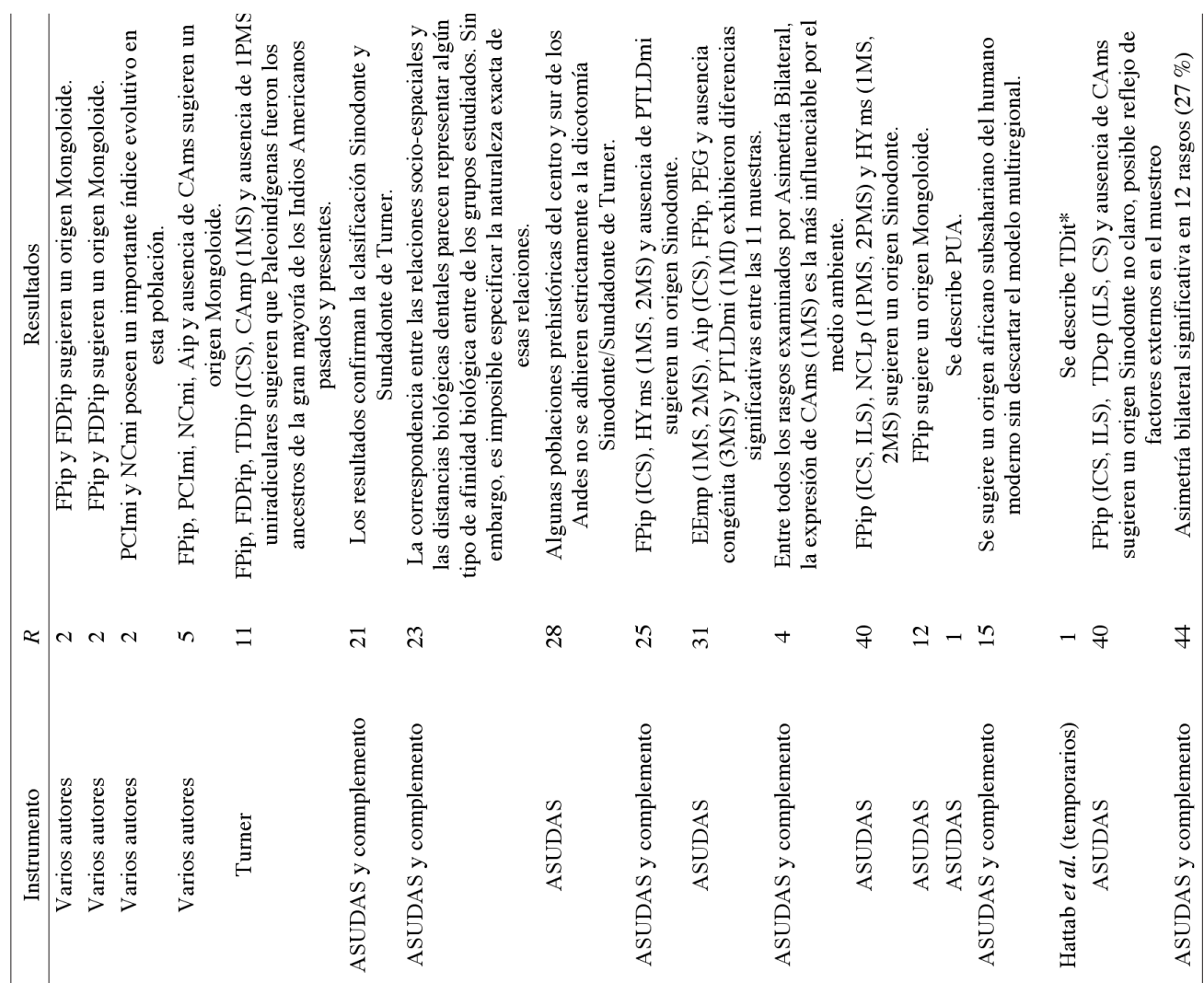

$q \geq-\frac{n}{a}-q$
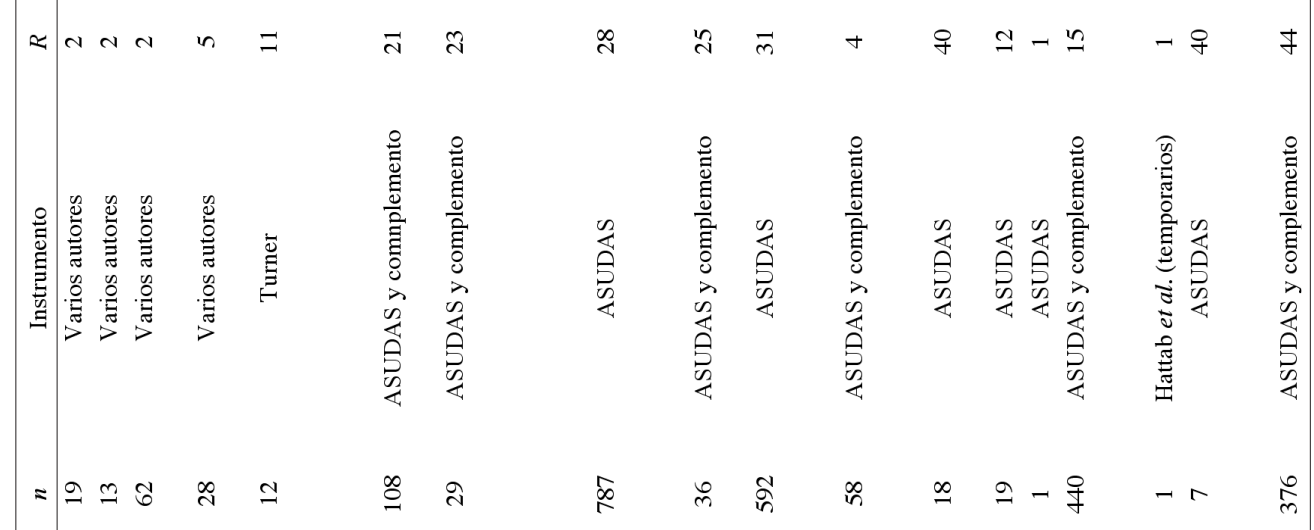

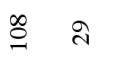

க)

$\stackrel{\infty}{2}-\frac{9}{+}-r$

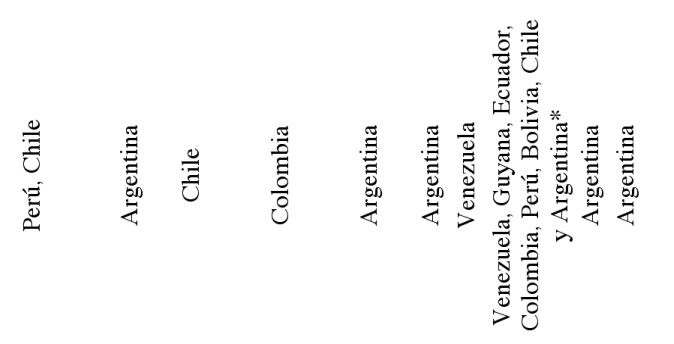

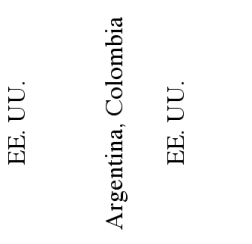
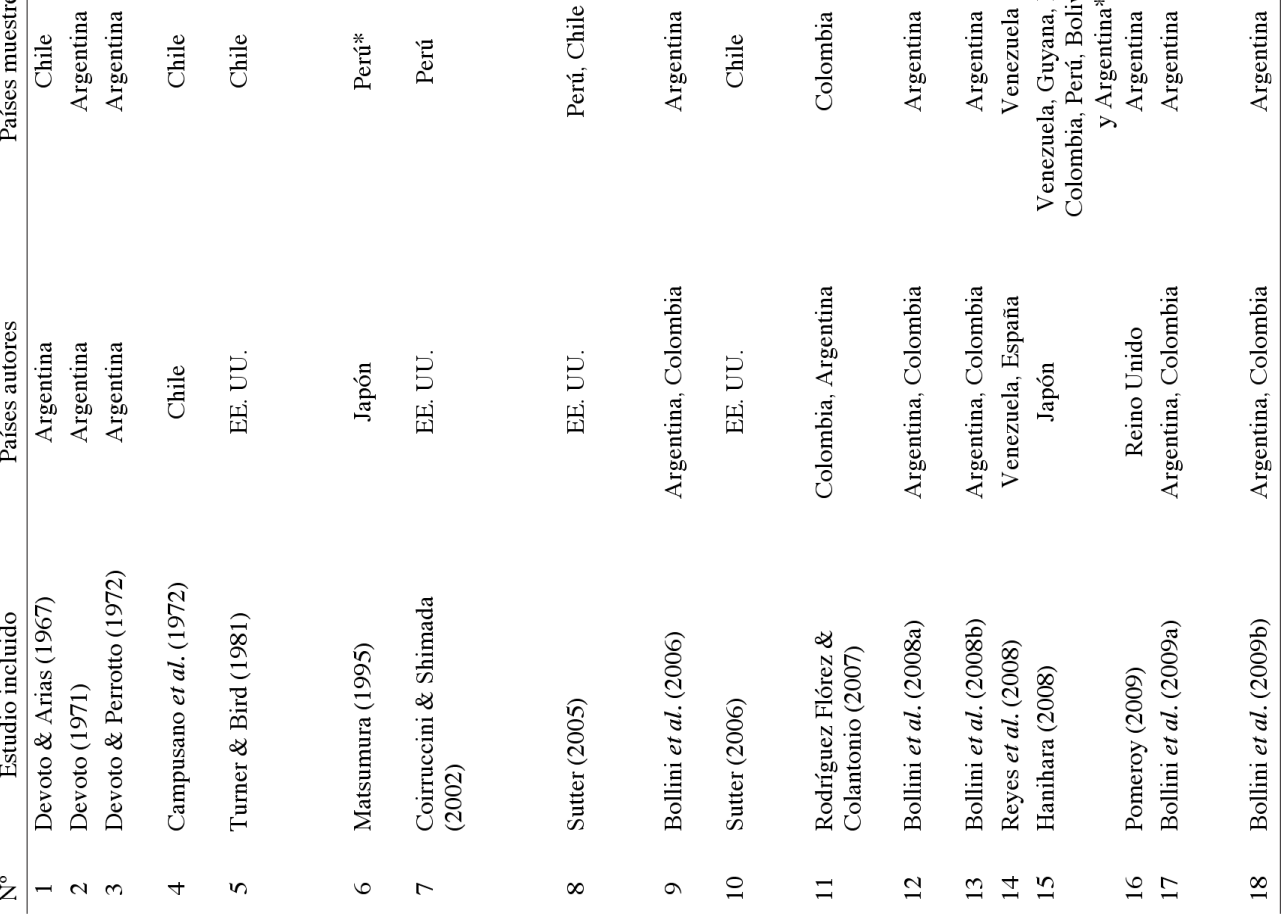

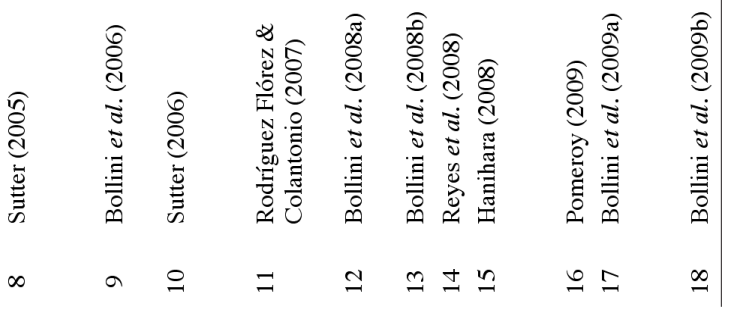




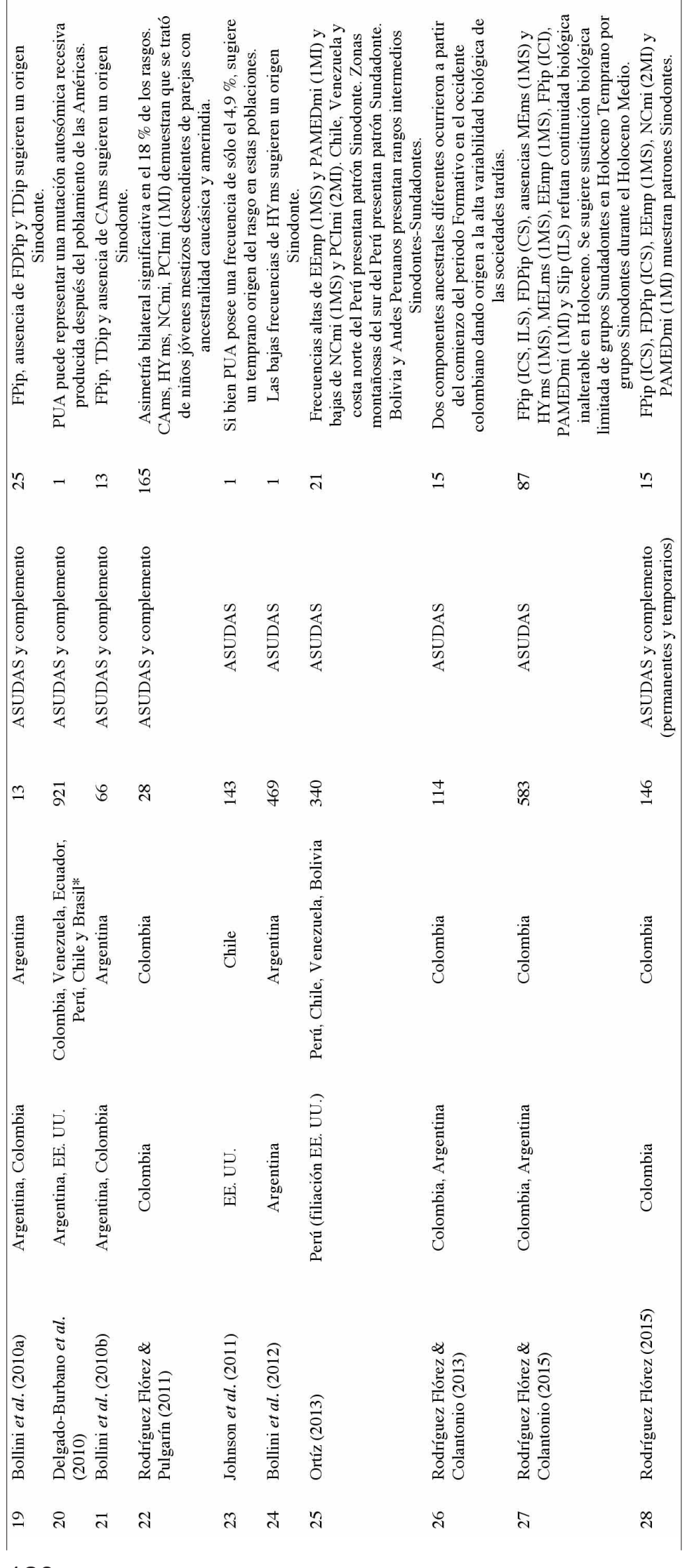

Outcomes. Desde las tempranas publicaciones de Devoto \& Arias, Devoto y Devoto \& Perrotto, haciendo el mayor hincapié sobre la expresión de los rasgos forma de pala, doble pala, y ausencia de tubérculo de Carabelli, los resultados de las investigaciones han orientado progresivamente a la sugerencia de una raíz mongoloide con origen Sinodonte. Por su parte, Sutter (2005) afirma con su estudio en muestras peruanas y chilenas que algunas poblaciones prehistóricas del centro y sur de los Andes no se adhieren necesariamente a la dicotomía Sinodonte/Sundadonte de Turner. Bollini et al., (2009a) encuentran en una muestra argentina que el origen Sinodonte no resulta del todo claro, probablemente debido a la influencia de factores externos en los registros. Rodríguez Flórez \& Colantonio (2013), analizando 87 rasgos en una población colombiana de 583 individuos, sugieren una sustitución biológica limitada de Sundadontes en el Holoceno temprano por grupos Sinodontes durante el Holoceno medio. Ese mismo año, Ortíz (2013) refiere la presencia de patrones Sinodontes en Chile, Venezuela y costa norte del Perú, Sundadontes en zonas montañosas del sur del Perú y rangos intermedios Sinodontes-Sundadontes en Bolivia y Andes peruanos (Tabla I).

Rodríguez Florez \& Colantonio (2007), Bollini et al. (2009b) y Rodríguez Flórez \& Pulgarín publican respectivos resultados sobre estudios de asimetría bilateral destacando la importancia de este tipo de análisis preliminar en muestras arqueológicas fragmentadas para subsecuentes interpretaciones y asignación de valor taxonómico poblacional (Tabla I).

\section{DISCUSIÓN}

La antropología dental, aunque controversial como especialidad antropológica, ha logrado evolucionar con técnicas, métodos y teorías propias aptas para resolver problemas de tipo histórico y socio-cultural (Rodríguez Flórez, 2003a, 2005). Al integrar la antropología, la odontología, la biología, la paleontología y la paleopatología, logra recuperar información evolutiva, biológica y cultural de las poblaciones pasadas y presentes mediante el análisis morfológico, métrico, patológico y modificatorio de los dientes (Moreno \& Moreno). Es así que las 
FONSECA, G. M.; ARAMBURÚ, G.; RODRÍGUEZ, I.; BOLLINI, G. A.; ATENCIO, J. P.; BERTA, M. J.; LÓPEZ-LÁZARO, S.; CANTÍN, M. \& LISSERA, R. G. Desarrollo de la investigación sobre variación morfológica de poblaciones históricas Sudamericanas utilizando rasgos dentales no métricos. Int. J. Morphol., 34(1):116-126, 2016.

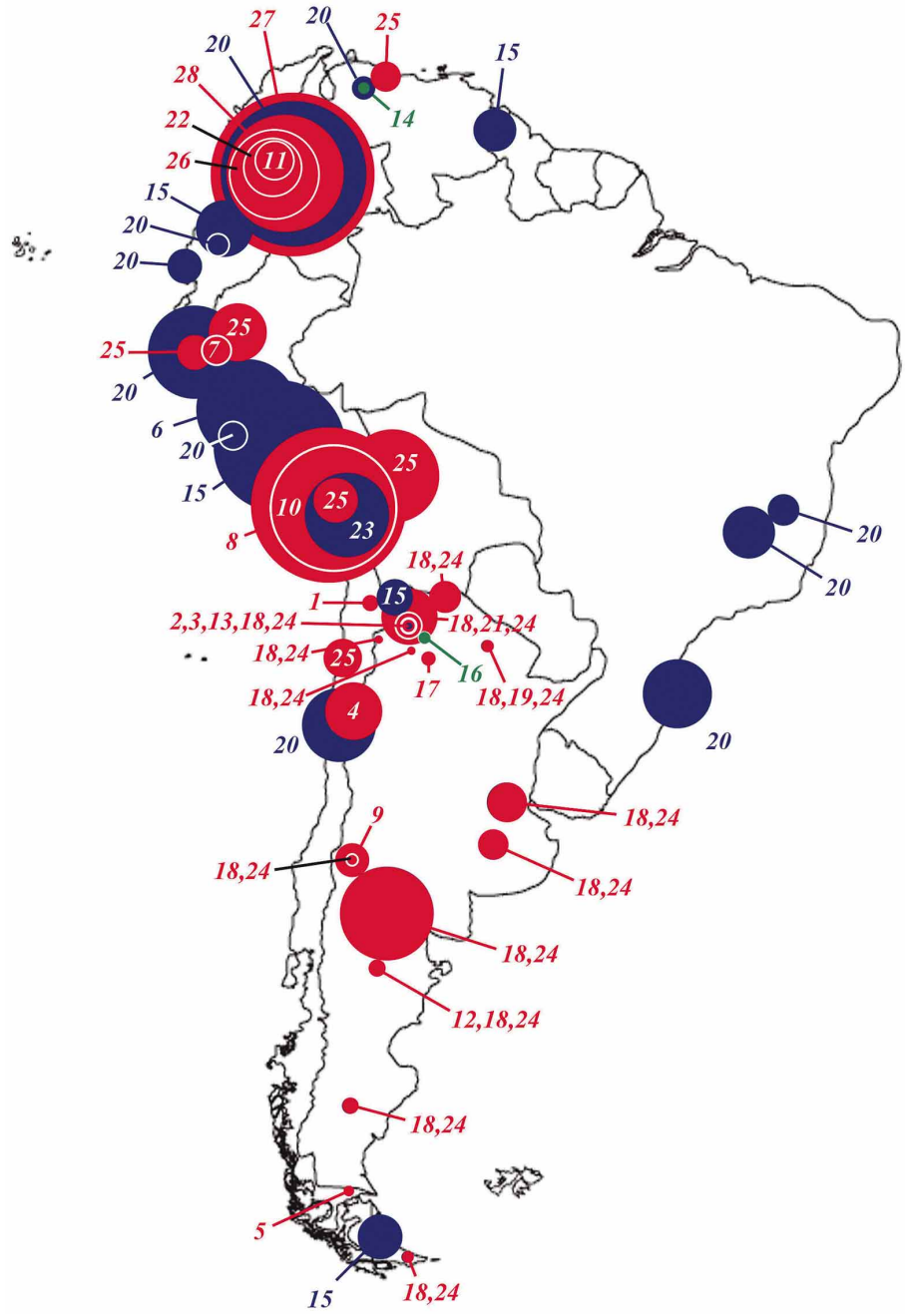

Fig. 2. Distribución geográfica de las poblaciones estudiadas por los artículos revisados (citas numéricas acordes a Tabla I). El tamaño de los círculos ha sido dispuesto proporcionalmente al tamaño de las muestras empleadas. En rojo los trabajos con muestreo exclusivo de poblaciones sudamericanas; en azul los trabajos asociados a muestreos globales; en verde los reportes de caso. Nótese la clara asimetría este-oeste en los estudios.

diferencias fenotípicas dentales encontradas entre dos o más grupos humanos a través del espacio y el tiempo pueden ser reconocidas como expresión de cambios en las frecuencias genéticas, y la comparación puede dar información relevante sobre el origen y los procesos de poblamiento en áreas específicas (Rodríguez Flórez, 2005). Mientras que genetistas y morfólogos han referido una escasa diversidad en las poblaciones sudamericanas, arqueólogos y lingüistas han argumentado que existen altos niveles de variabilidad cultural entre grupos del continente, esto especialmente relevante en la utilización de rasgos morfológicos dentales (Huffiman). Los aportes de Sutter (2005) refiriendo al menos dos eventos poblacionales ocurridos en Sudamérica (un primero Sundadonte protagonizado por paleoindios y descendientes del periodo Arcai- co-, seguido por un segundo Sinodonte -asociado con el aumento de fertilidad de poblaciones tempranas agricultoras-), representan un claro acercamiento a resolver origen y relaciones filogenéticas de los nativos americanos, "una de las áreas más controversiales de la arqueología y la antropología" (Lahr, 1995). El rasgo premolar Uto-Azteca ha sido propuesto como una variante única entre poblaciones recientes de nativos de Norteamérica y Sudamérica, no observado en el este asiático, distribución geográfica y temporal que ha sugerido diferencias entre poblaciones sudamericanas tempranas y tardías (Hufman), punto que coincidiría con las aserciones de Sutter (2005). Los hallazgos de Ortiz soportan además la teoría de una alta variabilidad entre diferentes grupos poblacionales sudamericanos cuando son comparados con series del este asiático. Recientes investigaciones sobre muestras de Perú, Chile, Venezuela y Brasil han sugerido que aún con elementos Sinodontes y Sundadontes, los prácticamente ausentes rasgos de Carabelli, cúspide 5 de molares maxilares, frecuencia intermedia de expresión de patrones de surcos intercuspídeos en Y, y las bajas frecuencias de primeros molares mandibulares con 3 raíces y aleteo, sugieren un patrón morfológico dental único para Sudamérica (Huffman). Evidentemente origen y poblamiento configuran tópicos de suma relevancia donde los análisis dentales han permitido establecer precisas relaciones biológicas y taxonómicas entre grupos (Rodríguez Flórez, 2005). Sin embargo, la abundancia de literatura no convencional en la disciplina y sus consecuentes limitaciones en la producción, difusión y acceso, configuran aún una dificultad insalvable al momento de intentar catalogar y revisar la información sobre el tema.

Esta literatura o documentación no convencional (gris, semi-publicada, invisible, menor o informal), corresponden a experiencias que no se difunden por los canales tradicionales de publicación comercial o en revistas no incluidas bases de datos, y que por tanto plantean problemas de acceso. Involucra documentos impresos de producción limitada y con poco tiraje, que no siguen necesariamente las nor mas de las ediciones tradicionales; con un contenido dirigido a lectores especializados, en lenguajes locales y no se ajusta a las normas de control bibliográfico (ISBN, ISNN o Indices de Impacto). Se estima que la literatura no convencional es aproximadamente el $10 \%$ de la información sobre un problema determinado (Manterola et al., 2013). En antropología, existe una enorme cantidad de información o reportes antropológicos inmensamente valiosos, que no 
FONSECA, G. M.; ARAMBURÚ, G.; RODRÍGUEZ, I.; BOLLINI, G. A.; ATENCIO, J. P.; BERTA, M. J.; LÓPEZ-LÁZARO, S.; CANTÍN, M. \& LISSERA, R. G. Desarrollo de la investigación sobre variación morfológica de poblaciones históricas Sudamericanas utilizando rasgos dentales no métricos. Int. J. Morphol., 34(1):116-126, 2016.

están en el dominio público debido a la necesidad de proteger los recursos culturales. Los departamentos académicos también tienen mucha literatura derivada de investigación llevada a cabo en todo el mundo, la cual sólo circula entre grupos académicos cerrados (tesis de pre y posgrado, actas de congresos, informes de investigación, memorias, proyectos, patentes, normas, traducciones científicas, documentos de sociedades científicas, boletines, cuadernos de trabajo, informes técnicos, etc.). Por otra parte, las leyes nacionales e internacionales, la naturaleza propietaria de los investigadores (miedo a que otros publiquen sus datos en bruto), y una serie de otras peculiaridades rodean este mandato de la práctica profesional antropológica. Así, las leyes que rigen el documento, la razón de que lo soliciten, el contenido del informe, y los planes del autor para su publicación son todos los factores de que afecten al acceso (Jahnke, 2016).

Esta misma necesidad de globalizar contenidos y normalizar la información ha tomado una gran fuerza en los instrumentos utilizados en antropología dental. Debido a la misma naturaleza de los rasgos morfológicos dentales, Turner et al. propusieron en 1991 un sistema clasificatorio basado en placas de referencia para cada rasgo denominado método ASUDAS. Los rasgos no métricos utilizados en este sistema son considerados generalmente no adaptativos, hipervariables, y relativamente comunes en las poblaciones de todo el mundo, lo que apoya la hipótesis de que los rasgos morfológicos dentales proporcionan información similar sobre afinidad biológica y estructura poblacional a la de los datos genéticos (Huf fman). Ha demostrado poseer un elevado potencial discriminatorio en los análisis de afinidades biológicas micro- y macroregionales (Cucina; Scott $\&$ Turner). Aunque el uso de estas placas de referencia es relativamente sencillo, las reglas publicadas por Turner et al. deben ser leídas y seguidas cuidadosamente, aún por experimentados observadores. El error interobservador debe ser minimizado en estudios comparativos (Scott \&Turner). Investigadores de al menos 36 países han utilizado ASUDAS, entre los que Argentina, Brasil y Chile componen los únicos sudamericanos en solicitar las placas entre 1985 y 2008 (con respectivas 2, 1 y 1 solicitudes), según citan Scott \& Turner. En esta revisión, aunque el método ASUDAS confirma su amplia inserción como instrumento estándar de valoración de rasgos a escala global -sugerencia realizada por los mismos Scott \&Turner-, aún continúa en deuda su difusión y reconocimiento una vez que se trascienden los límites de la disciplina. Sólo cinco de los 28 artículos revisados fueron publicados en revistas especializadas odontológicas (17,9\%), tres de ellos en el Journal of Dental Research (Devoto; Devoto \& Arias; Devoto y Perrotto) entre 1967-1972 (previos a la aparición de ASUDAS), uno en la Revista de la Facultad de Odontología de la Universidad de Antioquia en 2011 (Rodríguez
Flórez \& Pulgarín) y uno en el British Dental Journal el 2009, este último sin mención alguna a ASUDAS y con el rasgo designado fuera de la nómina aceptada mundialmente (Pomeroy). De igual modo, comparar tamaños muestrales desproporcionados puede no permitir un análisis confiable de los datos (Huf fman); se ha mencionado que el tamaño de las muestras es sumamente importante para la caracterización de colecciones esqueléticas procedentes de un sitio arqueológico; muestras de pocos individuos (menos de 30), usualmente son sesgadas por el efecto de variaciones azarosas de las mismas (Cucina; Rodríguez Cuenca, 2003). La Figura 2 resulta llamativa respecto a lo desproporcionado de los tamaños muestrales revisados y especialmente a la atención dedicada a algunos centros arqueológicos específicos. Los tres mayores tamaños muestrales han sido obtenidos en trabajos de colaboración internacional (DelgadoBurbano et al.) o reportes individuales (Sutter 2005, 2006), todos con filiación directa de los Estados Unidos (Tabla I).

En 1984, Ledergerber-de-kohli afirmaba que, aún contando América Latina con gran número de sitios históricos y arqueológicos de importancia, el estado de la ar queología de rescate no era satisfactorio debido a la falta de recursos técnicos y financieros, al número limitado de arqueólogos calificados y a la focalización de los estudios en sitios más prominentes. Si bien la autora hacía alusión a una forma especial de arqueología, la "de sitios amenazados por destrucción", resultan interesantes algunos conceptos vertidos por ella para explicar esa afirmación: la falta de legislaciones adecuadas, la poca comunicación entre profesionales e instituciones y la falta de cooperación entre los países del norte y del sur esto último considerando que los primeros están más avanzados en teoría arqueológica, métodos de excavación, técnicas de laboratorio, legislación y administración de sitios históricos. De igual modo, investigadores como Neves et al. (2007), señalan la complejidad de explicar la ausencia de estudios en contextos antropológicos y la relacionan con escasez de fondos.

Si bien podría afirmarse que los análisis antropológicos dentales sobre poblaciones históricas sudamericanas llevan un ritmo aceptable en calidad y cantidad, aún es poco lo que se conoce en cuanto a variabilidad de rasgos. Aunque Sudamérica, con respecto al norte y centro del continente, ha recibido la menor atención de la investigación antropológica, paradójicamente las más grandes colecciones de restos esqueléticos del Holoceno temprano y medio provienen de ella (Neves \& Hubbe, 2005). A esto se suma que la atención a la diversificación biológica de las poblaciones sudamericanas una vez migradas del este asiático ha sido mínima por no decir, ignorada (Huffman). 
FONSECA, G. M.; ARAMBURÚ, G.; RODRÍGUEZ, I.; BOLLINI, G. A.; ATENCIO, J. P.; BERTA, M. J.; LÓPEZ-LÁZARO, S.; CANTÍN, M. \& LISSERA, R. G. Desarrollo de la investigación sobre variación morfológica de poblaciones históricas Sudamericanas utilizando rasgos dentales no métricos. Int. J. Morphol., 34(1):116-126, 2016.

La manifiesta asimetría este/oeste expuesta en la Figura 2, refleja la evidente tendencia al estudio de poblaciones históricas andinas. Los restos culturales de los primeros grupos en asentarse están mejor representados por sitios en losAndes centrales, las llanuras del sur patagónico, Colombia, Perú, Chile y Brasil oriental, que en relación a otras vastas regiones prácticamente inexploradas -Ecuador, Bolivia, entre otras-, demuestra de algún modo, el estado de baja visibilidad para la investigación arqueológica de las últimas (Dillehay, 2008). Dicha situación, podría visualizarse desde diferentes puntos de vista: a) La inmensa geografía y diversidad ecológica de Sudamérica, particularmente en los Andes y en la cuenca del Amazonas, ha ofrecido opciones de movilización y búsqueda de recursos por parte de sus primeros pobladores. Este pequeño número de sitios en relación con el tamaño del continente puede reflejar la baja densidad poblacional, la reticencia de esos pobladores a explotar ciertas zonas ecológicas, así como también cuestiones elementales de preservación o incluso climáticas (Huffman); b) La región andina posee grandes tamaños poblacionales y altos niveles de flujo de genes en comparación con la no andina (Huf fman); c) Comparada con muchas otras partes del mundo, la región andina ofrece ventajas significativas para el investigador de los grupos étnicos prehistóricos, fundamentalmente su geografía y medio ambiente. Muchos de los fértiles valles insertos entre su comprimida geografía vertical, han favorecido el asentamiento de diferentes grupos étnicos cuya variedad ha proporcionado excelentes datos para estudios de variabilidad biológica. Particularmente los valles de montaña, han permanecido aislados del desarrollo moderno permitiendo la persistencia de prácticas culturales tradicionales. Aunque menos protegidos, los de la costa poseen climas en extremo áridos, lo que ha favorecido la conservación de una amplia gama de restos prehistóricos incluyendo artefactos en madera y hueso, textiles y hasta tejidos humanos y cabellos, todos fácilmente degradables por otras condiciones ambientales. Todo esto ha originado una evidente predilección de los antropólogos por examinar el origen étnico andino prehistórico durante, al menos, el último siglo (Reycraft, 2005).

Sin lugar a dudas, la presencia de países desarrollados en la colaboración museológica, sustentabilidad de proyectos, desarrollo y puesta a punto de metodologías, ha supuesto un avance significativo en los estudios de la antropología dental en Sudamérica (Bernal \& Luna; Huf fman; Ledergerber-de-kohli; Moreno \& Moreno), estos focalizados mayormente en las grandes y "más atractivas" urbes históricas (Matsumura; Corruccini \& Shimada, 2002; Sutter2005; Hanihara; Ortiz). Los países del área andina (Colombia, Ecuador, Perú, Bolivia y Chile), aunque presentan un desarrollo desigual, los aglutina el hecho de que parte de la población nativa ha sido sometida a estudios multinacionales en el marco de programas or ganizados y sustentados dada sus pertenencias a grandes unidades étnicas que comparten sus fronteras (Quechua y Aymará) (Rodríguez Cuenca, 1996). También es evidente que otros países sudamericanos han buscado posicionarse en la comunidad científica a partir de esfuerzos individuales, muchas veces discontinuados y dependientes de factores externos. La temprana influencia de A. Zoubov junto a los trabajos del antropólogo J. V. Rodríguez en Colombia (Moreno \& Moreno), enArgentina los mencionados estudios de Devoto, Arias y Perrotto -más allá de la falta de patrocinio, las reducidas líneas de investigación, la falta de actividad académica or ganizada y la sumisión a contextos sociopolíticos muchas veces poco acogedores- (Bernal \& Luna), o en Venezuela los adelantados aportes de Brewer Carias en 1964 (Reyeset al.) si bien muestran una actual vitalidad de las investigaciones, también expresan una necesaria implementación de políticas de patrocinio que permitan una mayor or ganización académica y labores de coordinación interinstitucional. La alianza Colombia-Argentina es un claro indicador de crecimiento.

Curioso es el caso de Brasil.Aunque bien los restos fósiles sudamericanos son relativamente pocos, existe una importante serie proveniente de Lagoa Santa (la reserva arqueológica más grande de ese país, en el Estado de Minas Gerais). Desde las primeros excavaciones de Lund a mediados del s. XIX hasta pasado 1970, al menos 250 esqueletos han sido recuperados por naturalistas y arqueólogos, tanto principiantes como profesionales; Neves \& Hubbe (2005) afirman que la mayoría de las excavaciones fueron reportadas en portugués en publicaciones locales, por lo que pocos antropólogos físicos norteamericanos y europeos tomaron conocimiento de estos yacimientos.Analizando esos fósiles con posterioridad, diferentes autores han identificado patrones no Mongoloides -lo que los asociaría muy cercanamente con recientes Australianos-, sugiriendo que las primeras poblaciones amerindias podrían ser reconocidas como "preMongoloides" (Lahr; Huffman). Nuevamente en el terreno de la literatura no convencional, un estudio de 2014 ha indicado la presencia de rasgos Sundadontes en poblaciones históricas de Lagoa Santa, Sambaquis (en las áreas costera del estado de Rio de Janeiro) y de nativos Botocudos (regionalmente pertenecientes a los actuales estados de Minas Gerais, Espírito Santo y Bahia) (Huf fman), hallazgo sumamente relevante pero imposible de catalogar en esta revisión. Solo el reporte de Delgado-Burbanoet al. sobre la presencia del premolar Uto-Azteca en poblaciones de Minas Gerais, Lagoa Santa y del norte y sur Sambaquí ha podido ser incluido. Rodríguez Cuenca (1996) afirma que Brasil está caracterizado por su especificidad en el desarrollo bioantropológico; al igual que Cuba y algunos países europeos, se ha formado académicamente en institutos de Biociencias y se le denomina por motivos administrativos 
FONSECA, G. M.; ARAMBURÚ, G.; RODRÍGUEZ, I.; BOLLINI, G. A.; ATENCIO, J. P.; BERTA, M. J.; LÓPEZ-LÁZARO, S.; CANTÍN, M. \& LISSERA, R. G. Desarrollo de la investigación sobre variación morfológica de poblaciones históricas Sudamericanas utilizando rasgos dentales no métricos. Int. J. Morphol., 34(1):116-126, 2016.

"biología humana". Dada la gran complejidad de variación poblacional aborigen, diferentes equipos multinacionales han abordado desde mediados del s. XX, análisis de polimorfismos genéticos, características demográficas, antropométricas, dermatoglíficas y afinidad lingüística cuyos resultados han incidido profundamente en el posterior desenvolvimiento de los estudios en biología humana de ese país (Rodríguez Cuenca, 1996). Si bien se han desarrollado ambiciosos proyectos sobre morfometría craneal de base arqueológica (Neves \& Hubbe, 2005), y nueva evidencia parece surgir apoyando los análisis de morfología dental en muestras actuales brasileñas para aplicación forense (Tnoco et al., 2016), los estudios de estos rasgos no métricos dentales en poblaciones históricas parecen aún estar en deuda, por lo menos en lo observado en esta revisión.

Se ha afirmado que existe en las últimas décadas una intensa popularización e internacionalización de la antropología biológica -evidente en el surgimiento de nuevas revistas y sociedades-, muy probablemente y entre otras razones, a la atención que ha despertado hacia la identificación de osamentas recientes con fines forenses, particular mente en áreas como la arqueología y la odontología forense. Esta tendencia se debe en parte a la situación ética y jurídica de movimientos de comunidades nativas y más especialmente, al descubrimiento de fosas comunes de desaparecidos por motivos políticos, todos problemas contemporáneos y de intensa realidad sudamericana (Rodríguez Cuenca, 1996; Rodríguez Flórez, 2005). Esto origina escenarios absolutamente diferentes donde las divisiones clásicas de los grandes grupos humanos (Caucasoides, Negroides y Mongoloides) hoy se ve reemplazada por una necesaria investigación regionalizada que logre contextualizar la ancestría con evolución, historia y fundamentalmente miscegenación; desafortunadamente, la gran mayoría de los estudios de morfología dental en todo el mundo no logran establecer los alcances y límites de sus muestras cuando se las pretende abordar desde un punto de vista forense, y aunque ASUDAS logra estandarizar cada uno de los rasgos, transpolar hallazgos museológicos o de etnias en particular con poblaciones modernas, urbanizadas y mixturadas -individuos vivos-, obliga a establecer convenientemente sus relaciones, variabilidad y valor probatorio en un proceso judicial (Tinoco et al.).

El análisis de rasgos morfológicos dentales permite caracterizar epigenéticamente poblaciones pretéritas y relacionarlas con otras anteriores o más recientes. El nivel de variabilidad en la expresión de los rasgos permite detectar procesos de miscegenación (hibridación, mestizaje) para reconstruir la historia biológica de poblaciones (tanto ar queológicas como actuales) y a la vez confirmar o rechazar la pertenencia étnica de un individuo encontrado en estado de esqueletización (Cucina). De igual manera, no es posible clasificar los grupos humanos del pasado sin considerar las relaciones locales establecidas entre la información biológica y cultural, lo que hace indispensable integrar la infor mación biológica en el plano interpretativo para complementar la comprensión de los grupos humanos prehispánicos sudamericanos (Rodríguez Flórez, 2003a). Esta revisión pone en evidencia que aunque el estudio de rasgos no métricos dentales permite observar relaciones con la ancestría, justificando su importancia en la identificación humana (Tinoco et al.) existe un grave desequilibrio (¿interés?) en la investigación de los diferentes países que componen el macizo de Sudamérica. Debe abogarse por un desempeño acor de no sólo con las innovaciones tecnológicas sino también con las necesidades sociales y económicas de los diferentes países sudamericanos. Coincidimos con Rodríguez Cuenca (1996) en la urgencia de estrechas lazos interdisciplinarios, institucionales e internacionales buscando la divulgación de los alcances de esta disciplina abandonando protagonismos y conformando asociaciones profesionales con reconocimiento ante organismos internacionales.

\section{AGRADECIMIENTOS}

Los autores agradecen a la Secretaría de Ciencia y Tecnología de la Universidad Nacional de Córdoba (S.E.C.y T.), Código de Incentivos 05/J146, resolución Nro. 203/14, Argentina. Además agradecen a la Dirección de Investigación, Universidad de La Frontera, Proyecto DIE15-0007.

FONSECA, G. M.; ARAMBURÚ, G.; RODRÍGUEZ, I.; BOLLINI, G. A.; ATENCIO, J. P.; BERTA, M. J.; LÓPEZLÁZARO, S.; CANTÍN, M. \& LISSERA, R. G. Development of research on morphological variation of historical SouthAmerican populations based on non-metric dental traits. Int. J. Morphol., 34(1):116-126, 2016.

SUMMARY: Studies of non-metric dental traits have managed to estimate biological relationships among ancient and contemporary human groups with a high taxonomic value. While South America has been subject of considerable research on settlement, migration and miscegenation, only a few studies have used information from dental traits for this purpose, with the consequent absence of data over wide geographical areas. A systematic review was conducted of the literature in MEDLINE, SciELO, REDALYC y LILACS, without limiting publication date. Full primary and secondary articles available in Spanish, English and Portuguese were included, where dental morphological traits were used to analyze SouthAmerican populations with a historical context prior to the twentieth century. The selected articles were evaluated by two researchers independently. The search revealed 
FONSECA, G. M.; ARAMBURÚ, G.; RODRÍGUEZ, I.; BOLLINI, G. A.; ATENCIO, J. P.; BERTA, M. J.; LÓPEZ-LÁZARO, S.; CANTÍN, M. \& LISSERA, R. G. Desarrollo de la investigación sobre variación morfológica de poblaciones históricas Sudamericanas utilizando rasgos dentales no métricos. Int. J. Morphol., 34(1):116-126, 2016.

2210 articles, of which 19 met the inclusion criteria, which added 9 after a complementary manual search. There is an unbalanced development of the SouthAmerican research, both in geographical focus where this is done, as countries and affliations of the authors. While there has been standardized instruments valuation of these traits, it is suggested to promote an interdisciplinary professionalization, the international support of its projects and the holistic approach of its content to enhance the applicability of their taxonomic value to contemporary populations.

KEY WORDS: Dental anthropology; Non-metric traits; South America; Forensic dentistry.

\section{REFERENCIAS BIBLIOGRÁFICAS}

Bernal, V. \& Luna, L. H. The development of dental research in Argentinean biological anthropology: current state and future perspectives. Homo, 62(5):315-27, 2011.

Bollini, G. A.; Atencio, J. P. \& Colantonio, S. E. El hipocono en poblaciones aborígenes de Argentina. Un análisis comparativo poblacional. Rev. Argent. Antropol. Biol., 14(1):57-64, 2012.

Bollini, G. A.; Rodríguez-Flórez, C. D. \& Colantonio, S. E. Dental nonmetric traits in a Pre-Conquest sample from Chubut Region of Patagonia, Argentina. Dent. Anthropol., 21(2-3):50-3, 2008a.

Bollini, G. A.; Rodríguez-Flórez, C. D. \& Colantonio, S. E. Dental non-metric traits in a Preconquest sample from Tastil Region in Argentina, South America. Bull. Int. Assoc. Paleodont., 2(1):1925,2008 b.

Bollini, G. A.; Rodríguez-Flórez, C. D. \& Colantonio, S. E. Dental non-metric traits in a Pre-Conquest sample "Calchaquí" fromArgentina, South America. Int. J. Morphol., 27(4):1063-7, 2009a.

Bollini, G. A.; Rodríguez-Flórez, C. D. \& Colantonio, S. E. Bilateral asymmetry in permanent dentition of 13 pre-conquest samples from Argentina (South America). Homo, 60(2):127$37,2009 b$.

Bollini, G. A.; Rodríguez-Flórez, C. D. \& Colantonio, S. E. Morfología dental en cráneos Toba de Argentina. Antropo, 21:27-38, 2010a.

Bollini, G. A.; Rodríguez-Flórez, C. D. \& Colantonio, S. E. Dental morphology in a sample of human skulls from Pampa Grande, Argentina. Int. J. Morphol., 28(3):685-96, 2010 b.

Bollini, G. A.; Rodríguez-Flórez, C.; Colantonio, S. E. \& Méndez, M. G. Morfología dental de una serie prehistórica deAraucanos provenientes de la Patagonia Argentina y su relación biológica con otras poblaciones prehistóricas argentinas y del mundoInt. J. Morphol., 24(4):705-12, 2006.

Campusano, C.; Figueroa, H.; Lazo, B.; Pinto Cisternas, J. \& Salinas, C. Some dental traits of Diaguitas Indian skulls. Am. J. Phys. Anthropol., 36(1):139-42, 1972.
Corruccini, R. S. \& Shimada, I. Dental relatedness corresponding to mortuary patterning at Huaca Loro, Peru. Am. J. Phys. Anthropol., 117(2):113-21, 2002.

Cucina, A. Morfología Dental. En: Cucina, A. (Ed.) Manual de Antropología Dental. Mérida, UniversidadAutónoma de Yucatán, 2011.

Delgado-Burbano, M. E.; Scott, G. R. \& Turner, C. G. 2nd. The Uto-Aztecan premolar among North and South Amerindians: Geographic variation and genetics. Am. J. Phys. Anthropol., 143(4):570-8, 2010.

Devoto, F. C. Shovel-shaped incisors in Pre-Columbian T astilian Indians. J. Dent. Res., 50(1):168, 1971.

Devoto, F. C. \&Arias, N. H. Shovel-shaped incisors in earlyAtacama Indians. J. Dent. Res., 46(6):1478, 1967.

Devoto, F. C. \& Perrotto, B. M. Groove pattern and cusp number of mandibular molars from Tastilian Indians. J. Dent. Res., 51(1):205, 1972.

Dillehay, T. D. Profiles in Pleistocene History. En: Silverman, H. \& Isbell, W. H. (Eds.). The Handbook of South American Archaeology. New York, Springer, 2008. pp.29-43.

Hanihara, T. Morphological variation of major human populations based on nonmetric dental traits. Am. J. Phys. Anthropol., 136(2):169-82, 2008.

Hernández, J.; Villavicencio, J.; Arce, E. \& Moreno, F . Talón Cuspídeo: reporte de cinco casos. Rev. Fac. Odontol. Univ. Antioq., 21(2):208-17, 2010.

Huffman, M. Biological Variation in South American Populations using Dental Non-Metric Traits: Assessment of Isolation by Time and Distance. Tesis Doctoral. Columbus, Ohio State University 2014.

Jahnke, L. Anthropology Research Guide. Emory University Libraries, 2016. Disponible en: http:// guides.main.library.emory.edu/anthropology_research

Johnson, K. M.; Stojanowski, C. M.; Miyar, K. O.; Doran, G. H. \& Ricklis, R.A. New evidence on the spatiotemporal distribution and evolution of the Uto-Aztecan premolar . Am. J. Phys. Anthropol., 146(3):474-80, 2011.

Lahr, M. M. Patterns of modern human diversification: Implications for Amerindian origins. Am. J. Phys. Anthropol., 38 (Suppl. 2):163-98, 1995.

Ledergerber-Kohli, P. Planteamientos para promover el desarrollo de la arqueología de rescate en América Latina. Bol. Antropol. Am., (10):109-17, 1984

Manterola, C.; Astudillo, P.; Arias, E.; Claros, N. \& Grupo MINCIR. Systematic reviews of the literature: what should be known about them. Cir. Esp., 91(3):149-55, 2013.

Matsumura, H. Dental characteristics affinities of the Prehistoric to Modern Japanese with the East Asians, American Natives and Australo-Melanesians. Anthropol. Sci., 103(3):235-61, 1995. 
FONSECA, G. M.; ARAMBURÚ, G.; RODRÍGUEZ, I.; BOLLINI, G. A.; ATENCIO, J. P.; BERTA, M. J.; LÓPEZ-LÁZARO, S.; CANTÍN, M. \& LISSERA, R. G. Desarrollo de la investigación sobre variación morfológica de poblaciones históricas Sudamericanas utilizando rasgos dentales no métricos. Int. J. Morphol., 34(I):116-126, 2016.

Matsumura, H. \& Hudson, M. J. Dental perspectives on the population history of Southeast Asia. Am. J. Phys. Anthropol., 127(2):182$209,2005$.

Moreno, S. \& Moreno, F. Importancia clínica de la antropología dental. Rev. Estomatol., 15(2) Supl. 1:42-53, 2007.

Neves, W. A. \& Hubbe, M. Cranial morphology of early Americans from Lagoa Santa, Brazil: implications for the settlement of the New World. Proc. Natl. Acad. Sci. USA, 102(51):18309-14, 2005.

Neves, W. A.; Hubbe, M. \& Correal, G. Human skeletal remains from Sabana de Bogotá, Colombia: a case of Paleoamerican morphology late survival in South America? Am. J. Phys. Anthropol., 133(4):1080-98, 2007.

Ortiz, A. Dental morphological variation among six Pre-Hispanic South American populations with implications for the peopling of the New World. Dent. Anthropol., 26(1-2):20-32, 2013.

Pomeroy, E. Labial talon cusps: a South American archaeological case in the deciduous dentition and review of a rare trait. $B r$. Dent. J., 206(5):277-82, 2009.

Reyes, G.; Padilla, A.; Palacios, M. \& Bonomie, J. Posible presencia del rasgo dental premolar "Uto-Azteca" en un cráneo de época prehispánica (siglos II a.C., siglo IV d.C.), cementerio de "Las Locas", Quíbor (Estado Lara, Venezuela). Bol. Antropol., 26(72):53-85, 2008.

Reycraft, R. M. (Ed.). Us and Them: Archaeology and Ethnicity in the Andes. Los Angeles, The Cotsen Instituye of Archaeology, University of California, 2005. pp.1-11.

Rodríguez Cuenca, J. V. Panorama de la Antropología biológica en Colombia y su relación con el ámbito latinoamericano y mundial. Maguaré, 11-12:75-102, 1996.

Rodríguez Cuenca, J. V. Dientes y Diversidad Humana: Avances de la Antropología Dental. Bogotá, Editora Guadalupe Ltda., 2003. pp.43-76.

Rodríguez Flórez, C. D. Antropología dental en Colombia. Comienzos, estado actual y perspectivas de investigaciónAntropo, 4:1727, 2003a.

Rodríguez-Flores, C. D. Antropología Dental Prehispánica: Variación y Distancias Biológicas en la Población Enterrada en el Cementerio Prehispánico de Obando, Valle del Cauca, Colombia entre los Siglos VIII y XIII d.C. Miami, Syllaba Press, 2003b.

Rodríguez-Flórez, C. D. La antropología dental y su importancia en el estudio de los grupos humanos. Rev. Fac. Odontol. Univ. Antioq., 16(1-2):52-9, 2005.

Rodríguez-Flórez, C. D. Non-metric variation of the Muisca dentition in the northern Andes of Colombia, South America. Bull. Int. Assoc. Paleodont., 9(1):17-28, 2015.

Rodríguez-Flórez, C. D. \& Colantonio, S. E. Bilateral asymmetry of upper permanent dentition in six archaeological pre-conquest samples from Colombia, South America. Dent. Anthropol., 20(2):19-23, 2007.
Rodríguez-Flórez, C. D. \& Colantonio, S. E. Tumbas, dientes y cultura: 2.500 años de microevolución y los orígenes de las sociedades prehispánicas en la región arqueológica calima de Colombia, Sur América. Antropo, 30:13-32, 2013.

Rodríguez Flórez, C. D. \& Colantonio, S. E. Biological affinities and regional microevolution among pre-Hispanic communities of Colombia's NorthernAndes. Anthropol. Anz., 72(2):141-68, 2015.

Rodríguez-Flórez, C. D. \& Pulgarín, E.Asimetría dental e identificación de mestizaje en una muestra poscolonial desenterrada en la Catedral de Pereira, Colombia.Rev. Fac. Odontol. Univ. Antioq., 22(2):139-52, 2011.

Scott, G. R. \& Turner II, C. G. History of Dental Anthropology. En: Irish J. D. \& Nelson, G. (Eds.). Technique and Application in Dental Anthropology. Cambridge, Cambridge University Press, 2008.

Sutter, R. C. The prehistoric peopling of South America as inferred from epigenetic dental traits. Andean Past, 7:183-217, 2005.

Sutter, R. C.The test of competing models for the prehistoric peopling of the Azapa Valley, Northern Chile, using matrix correlations. Chungara Rev. Antropol. Chil., 38(1):63-82, 2006.

Tinoco, R. L.; Lima, L. N.; Delwing, F, Francesquini, L. Jr \& Daruge, E. Jr. Dental anthropology of a Brazilian sample: Frequency of nonmetric traits. Forensic Sci. Int., 258:102.e1-5, 2016.

Turner, C. G. \& Bird, J. Dentition of Chilean paleo-Indians and peopling of the Americas. Science, 212(4498):1053-5, 1981.

Turner, C. G. II; Nichol, C. R. \& Scott, G. R.Scoring Procedures for Key Morphological Traits of the Permanent Dentition: the Arizona State University Dental Anthropology System. En: Kelley, M. A. \& Larsen, C. S. (Eds.). Advances in Dental Anthropology. New York, Wiley-Liss, 1991. pp.13-31.

Dirección para Correspondencia:

Gabriel M. Fonseca, DDS, Ph.D.

Facultad de Odontología

Universidad de La Frontera

Avenida Francisco Salazar 01145

Temuco

CHILE

Email: gabriel.fonseca@ufrontera.cl 